\title{
Is Cardiomyopathy Associated with Peripartum or Anaphylactic Reaction? Which One?
}

\begin{abstract}
Peripartum cardiomyopathy (PPCM) is a form of dilated cardiomyopathy of unclear etiology. Cardiac failure developes in the last month of pregnancy or within five months after delivery. Symptoms and laboratory findings of PPCM are not specific and transthoracic echocardiography (TTE) is the most important tool in diagnosis. Anaphylactic reaction treated with epinephrine due to blood transfusion may considered as a reason of cardiomyopathy. Here we reported a 21 year-old cardiomyopathy patient presented with pulmonary edema resolved with diuretic treatment. Anaphylaxis reaction was observed in patient after blood transfusion and the patient was treated with epinephrine.
\end{abstract}

Keywords: Peripartum cardiomyopathy; Anaphylactic blood transfusion reaction; Intensive care unit; Cardiogenic pulmonary edema

\section{Introduction}

Peripartum cardiomyopathy (PPCM) is a form of dilated cardiomyopathy of unclear etiology, defined as heart failure secondary to left ventricular systolic dysfunction [1,2]. The exact pathogenetic mechanism is not fully understood. Several pathogenetic factors are inflammatory, infectious, genetic, autoimmune, oxidative stress [2]. Risk factors include multiparity, black race, older maternal age, preeclampsia, and gestational hypertension. Transthoracic echocardiography (TTE) is the most important tool for diagnostic confirmation or exclusion of PPCM and should be performed in every suspected case [2]. The approach for treating PPCM mirrors that of treating congestive heart failure.

Anaphylaxis is a severe systemic response by allergen mediated type 1 reaction(decreased systemic vascular resistance, hypotension, increased pulmonary vascular resistance, decreased left ventricular preload) initiated by exposure to a specific antigen in a sensitized organism [3]. Rare cases have been reported in the literature about cardiomyopathy in the setting of anaphylaxis treated with epinephrine [4,5]. But PPCM, which may be associated with anaphylaxis and epinephrine administration, has never been reported. Here, we reported a PPCM patient presented with pulmonary edema with anaphlaxis and epinephrine administration after blood transfusion.

\section{Case Presentation}

Twenty one yearold woman was admitted to the department of obstetrics and gynecology at gestastional age of 37 weeks with bleeding. The patient had no previous history of cardiac or pulmonary disease. She was a housewife, gravida 1, parity 0 . Her pregnancy was terminated by cesarean section due to placenta ablation and intrauterine ex fetus. She was taken to the intensive care unit after operation. The glaskow coma score (GCS) was 14 and physical examination was normal on first

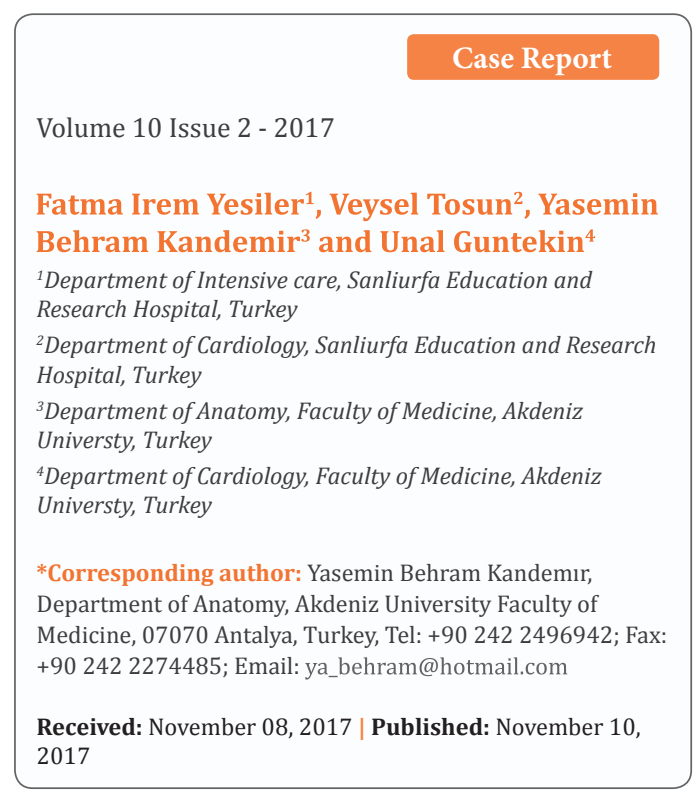

examination, but the hemoglobin value decreased to $7.4 \mathrm{~g} / \mathrm{dl}$. Erythrocyte suspension was transfused forbleeding and low postoperative hemoglobin value. An anaphylactic reaction developed in the patient during first blood transfusion (after about $100 \mathrm{ml}$ ). Deep hypotension, bilateral diffuse bronchospasm andanasarca edemawere observed in patient. Adrenaline (1:10000) was administered intravenously to the patient andinvasive blood pressure monitoring was performed.On first assessment laboratory findings were hemoglobin: 7,4 g/dl, hematocrit: $23 \%$, platelet: 75000 , creatinine: $1,19 \mathrm{mg} / \mathrm{dl}$, lactate dehydrogenase (LDH): $508 \mathrm{U} / \mathrm{L}$, calcium: 6,6 mg/dl. Following hemoglobin was $5,5 \mathrm{~g} / \mathrm{dl}$ thus 3 units of washed erythrocyte suspension were transfused to the patient. The patient complained of dyspnea, orthopnea and lower limb edemaafter 3 hours from blood transfusion. Oxygen saturation was $84 \%$ in room air. On auscultation, bilateral inspiratory rales were heard at all zones of the lungs and she had bilateral marked pretibial oedema. Four liters/minute ofnasal oxygen, diuretic (furosemide intravenous) and anticoagulant (enoxaparin 6000 unit 2x1 subcutaneously) were given with predicted pulmonary edema and pulmonary thromboembolism. Her arterial blood gas analysis showed $\mathrm{pH}$ : 7,52; $\mathrm{pO}_{2}:$ 69,6 mmHg; $\mathrm{pCO}_{2}$ : 36,2 $\mathrm{mmHg} ; \mathrm{HCO}_{3}: 29,2 \mathrm{mmol} / \mathrm{L}$; $\mathrm{SO}_{2}: 86 \%$. Bilateral infiltrations especially at the middle and lower zones and cardiomegaly were notable on the chest x-ray (Figure 1). The bedside transthoracic echocardiography showed normal right ventricles, pulmonary artery pressure of $30 \mathrm{mmHg}$, left ventricular EF of $40 \%$ by Teicholz and Simpson'a methods 
(Figure 2). The case was diagnosed with PPCM and she was given treatment consisting of an angiotensin converting enzyme inhibitör (ACEI) and furosemide. Regression was observed on control chest X-ray two days later (Figure 3). When the clinical state findings improved, she was transferred to Department of Obstetrics and Gynecology. The patient was discharged after 5 dayswith ACEI, furosemide and metoprolol.

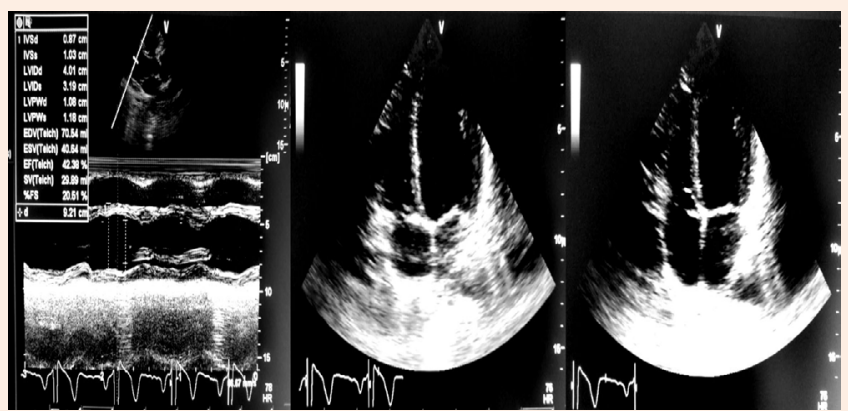

Figure 1: The chest x-ray. A chest x-ray shows bilateral infiltrations at the middle and lower zones, and cardiomegaly consistent with pulmonary edema.

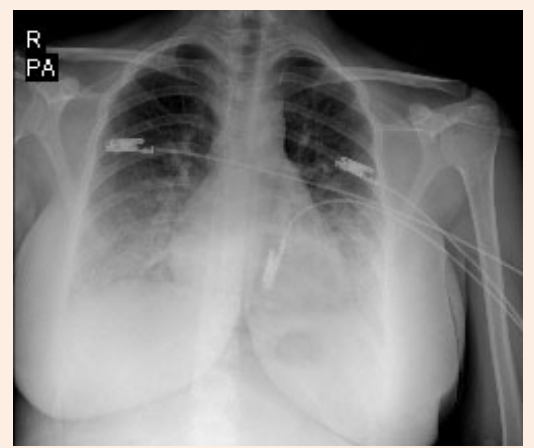

Figure 2: The M-Mode transthoracic echocardiography. A M-Mode transthoracic echocardiography shows a calculated left ventricular EF of $40 \%$.

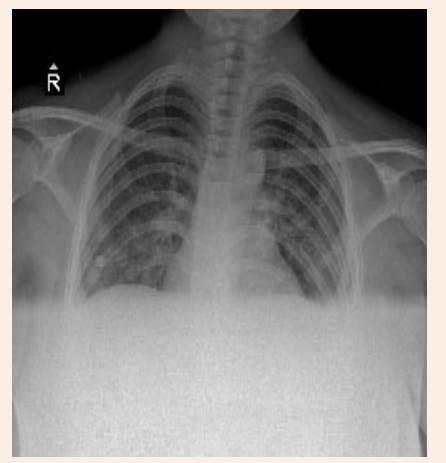

Figure 3: The control chest x-ray. A control chest x-ray shows bilateral regression of infiltrations.

\section{Discussion}

PPCM is a rare form of heart failure with unknown etiology, which affects previously healthy women in late pregnancy or early puerperium. The diagnosis is defined on the basis of four criteria; 1) Development of cardiac failure in the last month of pregnancy or within five months of delivery, 2) Absence of an identifiable cause for the cardiac failure, 3) Absence of recognisable heart disease prior to the last month of pregnancy, 4) LV systolic dysfunction demonstrated by classic echocardiographic criteria such as decreased shortening fraction or ejection fraction (EF) $[2,6]$. Symptoms and laboratory findings of PPCM are not specific. TTE is the gold standard in diagnosis. Our patient presented with pulmonary edema that resolved with diuretic therapy. PPCM was diagnosed in our case due to no stories of heart diseaseuntill acute onset of pulmonary edema, occurrence of the disease in the early postpartum period, no other reasons to explain heart failure and presence of decreased EF in TTE.There was no prior history of heart failure in the hospital's system registry. Also, at postpartum third month, an increase in left ventricular EF to $60 \%$ was observed with effective therapy of beta blocker, ACEI and diuretic. Therefore, a pre-existing dilated cardiomyopathy or other causes of chronic heart failure could not be considered.

Although the etiology of PPCM remains unclear, a number of potential risk factors have been proposed previously: mulitparity, women of African descent, age greater than 30 years, pregnancy with multiple foetuses, a history of preeclampsia, eclampsia or postpartum hypertension, maternal cocaine abuse or selenium deficency, long-term ( $>4$ weeks) oral tocolytic therapy with adrenergic agonists, inflammation, viral infections, autoimmunity, stress-active cytokines and genetic factors [2,7]. In literature, there are rare case reports about cardiomyopathy following epinephrine administration for anaphylaxisbut, no evidence of anaphylactic or epinephrine administration that may be the cause of PPCM was observed [4,5]. In our case, anaphylactic reaction treated with epinephrine due to blood transfusion mayconsidered as a reason of cardiomyopathy. More case series are needed in this regard. Anaphylactic reaction associated cardiomyopathy is a different phenomenon that causes cardiomyopathy but, in this case we did not considered because of the patient had not hypereosinophilia on blood tests.

Patients with PPCM should be managed with established treatment for acute heart failure with oxygen, intravenous diuretics, ACEI with intravenous nitrates and inotropes if required. Beta adrenergic blockers should be added when acute heart failure has been stabilised. Patients with a reduced EF have a higher thrombotic risk.Therefore, low-molecular weight heparin should also be administered. Diuretic, ACEI, anticoagulant therapy and beta blockers after stabilizationwere started to our patient $[1,2]$.

Some studies showed high mortality rate such as $15 \%$ or $32 \%$ at 6 months [8]. Survivors of PPCM have 30\% risk of relapse and significant decrease of left ventricular function in subsequent pregnancies with reported 55\% mortality and morbidity rates associated with recurrence in subsequent pregnancy [9]. So, follow-up and treatment should be performed in the intensive care unit after diagnosis.

\section{Conclusion}

PPCM is a disease with high rates of fetal-maternal morbidity and mortality, whose symptoms are nonspecific, whose diagnosis 
is based on the exclusion of other possible causes.Therefore, the clinicians should be aware of this disease. Although the etiology is not fully known, it should be evaluated well about risk factors. PPCM can be diagnosed quickly with bedside transthoracic echocardiography, follow-up and treatment in the intensive care unit can improve the prognosis.

\section{Conflict of interest}

None declared.

\section{Financial Disclosure}

The authors declared that this study has received no financial support.

\section{References}

1. Silwa K, Hilfiker-Kleiner D, Petrie MC, Mebazaa A,Pieske B, etal.(2010) Current state of knowledge on etiology, diagnosis,management, and therapy of peripartum cardiomyopathy: a position statement from the Heart Failure Association of the European Society of Cardiology Working Group on peripartum cardiomyopathy. Eur J Heart Fail 12(8): 767-778.

2. Kleiner HD, Haghikia A, Nonhoff J, Bauersachs J (2015) Peripartum cardiomyopathy: currentmanagement and future perspectives. Eur Heart J 36(18): 1090-1097.
3. Hartmann M, Bode C, Zirlik A (2008) Anaphylactic shock-associated cardiomyopathy. Int J Cardiol 127(3): 136-137.

4. Magri CJ, Fava S, Felice H (2011) Inverted takotsubo cardiomyopathy secondary to adrenaline injection. Br J Hosp Med (Lond) 72(11): 646-647.

5. Khoueiry G, Abi Rafeh N, Azab B, Markman E, Waked A, et al. (2013) Reverse Takotsubo cardiomyopathy in the setting of anaphylaxis treated with high-dose intravenous epinephrine. J Emerg Med 44(1): 96-99.

6. Giverts MM (2013) Peripartum Cardiomyopathy. Circulation 127(20): 622-626.

7. Elkayam U, Akhter MW, Singh H, Khan S, Bitar F, et al. (2005) Pregnancy-Associated Cardiomyopathy: Clinical characteristics and a comparison between early and late presentation. Circulation 111(16): 2050-2055.

8. Duncker D, Bauersachs J, Hilfiker-Kleiner D, Veltmann C (2015) Underestimated risk for life-threatening ventricular arrhythmias in newly diagnosed peripartum cardiomyopathy? Eur Heart J 36(18): 1067-1068.

9. Wang M (2015) Peripartum cardiomyopathy: case reports. Perm J 13(4): 42-45. 